\title{
Scattering and Doppler Spectral Analysis for a Fast-Moving Target above Time-Varying Lossy Dielectric Sea Surface
}

\author{
Ke Li, Lixin Guo, and Juan Li \\ School of Physics and Optoelectronic Engineering, Xidian University, Xian 710071, China \\ Correspondence should be addressed to Ke Li; xidian_keli@163.com
}

Received 7 July 2016; Revised 12 September 2016; Accepted 20 September 2016

Academic Editor: Paolo Burghignoli

Copyright ( $) 2016 \mathrm{Ke} \mathrm{Li} \mathrm{et} \mathrm{al.} \mathrm{This} \mathrm{is} \mathrm{an} \mathrm{open} \mathrm{access} \mathrm{article} \mathrm{distributed} \mathrm{under} \mathrm{the} \mathrm{Creative} \mathrm{Commons} \mathrm{Attribution} \mathrm{License,} \mathrm{which}$ permits unrestricted use, distribution, and reproduction in any medium, provided the original work is properly cited.

\begin{abstract}
A numerical electromagnetic method based on the physical optics with physical optics method (PO-PO) is employed to calculate backscattered returns from a missile-like target above sea surface. Surfaces are time-varying Monte Carlo simulations initialized as realizations of a Pierson-Moskowitz spectrum. The monostatic normalized radar cross section of composite model by the hybrid PO-PO method is calculated and compared with those by the conventional method of moments, as well as the runtime and memory requirements. The results are found to be in good agreement. The runtime shows that the hybrid PO-PO method enables largescale time-varying Monte Carlo simulations. The numerical simulations of the Doppler spectrum from the fast-moving target above time-varying lossy dielectric sea surface are obtained, and the Doppler spectra of backscattered signals from this model are discussed for different incident angles, speed of flying target, wind speeds, incident frequencies, and target altitudes in detail. Finally, the coupling effects on Doppler spectra are analyzed. All the results are obtained at the incidence of horizontal polarization wave in this study.
\end{abstract}

\section{Introduction}

Doppler spectra are important in many fields ranging from target detection over sea surface, remote sensing at sea, and extraction of wave parameters from simulated and measured radar sea echoes [1-3]. Doppler spectra analysis depends on wind speed and radar parameters for a time-varying sea surface. The Doppler shift is related to a power-weighted mean velocity of the moving scatterer. Moreover, the Doppler bandwidth is determined by the variance of the velocity distribution of the scatter units on the scatterer [4]. When a moving target is located above sea surface, the signals from the target may be submerged by the signals from sea clutter. Therefore, the Doppler spectrum analysis of the composite model seems promising. In general, Monte Carlo Doppler simulations from time-evolving surfaces are more computationally demanding than common scattering calculations from time-frozen surfaces, and they are more challenging in Doppler spectral simulations of electrical large-scale targets in actual sea background because of their large computer memory and huge computational cost [5].
With the development of computational electromagnetics, many EM methods, including numerical and analytical methods, have been proposed to calculate the scattering from time-varying rough sea surface and targets in rough sea surface backgrounds. Recently, Guo et al. [6] proposed the EPILE method to analyze scattering from targets above or below rough surfaces. Qi et al. [7] employed a hybrid iterative method that combined KA and MLFMA to solve the EM backscattering from rough sea surface and targets. Liu and Jin [8] developed the FEM with domain decomposition to study the Doppler spectrum of a flying target above dynamic oceanic surfaces. Toporkov and Brown [9] combined the MOMI with the fast multipole method to address the Doppler spectra of sea surfaces at L-band. Hybrid methods combining analytical methods with numerical algorithms have greatly improved computational efficiency in solving typical composite scattering problems. Previously, the iterative method seemed to be the most appropriate in dealing with the large memory requirement and large runtime Doppler spectral simulation problems, because the iterative method allows EM scattering calculation from the target and sea surface 


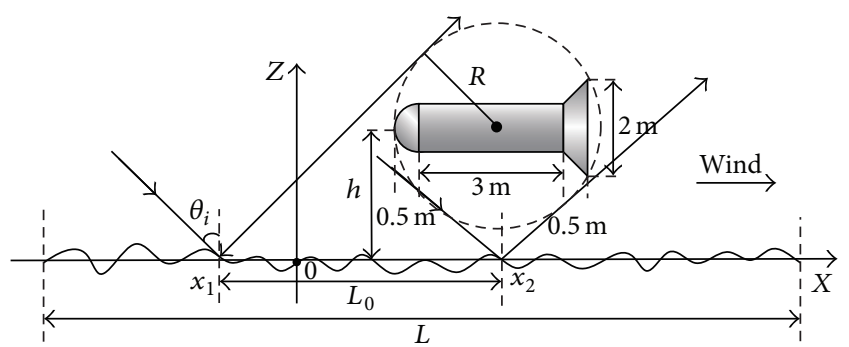

FIGURE 1: Scattering of the composite model.

separately $[10,11]$ and then solves the mutual EM coupling between those two parts using an iterative process.

In our previous works, the hybrid PO-PO method [1215] was proposed to simulate the composite scattering of a perfectly conducting (PEC) cylinder above the random rough surface. The PO-PO method uses an iterative process, in which both the scattering of a target and the underlying rough surface are calculated by the PO method, and the interactions between them are solved by the Huygens equivalence principle and multipath scattering strategy. In this paper, the local-coupling area was utilized to reduce the total-coupling area when the hybrid PO-PO method was implemented. The monostatic normalized radar cross section (NRCS) of composite model by the hybrid PO-PO method was calculated and compared with that by the conventional method of moments (MOM) [16]. The numerical simulations of the Doppler spectrum from the PEC fast-moving missilelike target above time-varying lossy dielectric sea surface were then obtained, and the Doppler spectra of backscattered signals from such simulations were discussed from different incident angles, frequencies, and target altitudes in detail. In this paper, all fields and currents were assumed to have a time-harmonic dependence of the form $e^{-i \omega t}$, and the horizontally ( $y$-direction) polarized (TE) wave incidence $\left(E_{i y}\right)$ is discussed.

\section{Composite Scattering Model}

2.1. Sea Surface Model. We will consider linear onedimensional (1D) lossy dielectric random rough surfaces. The time-evolving surface has an arbitrary profile and was generated by using the spectral method. The spectral method had been described by Toporkov and Brown [9] in detail, and the surface profile is assumed to be a superposition of harmonics whose amplitudes are independent of Gaussian random variables with variances proportional to a surface roughness spectrum. According to the procedures described by Rino et al. [17], and considering a 1D directional sea spectrum proposed by Pierson Jr. and Moskowitz [18], the $1 \mathrm{D}$ lossy dielectric random rough surface generated by the Monte Carlo realization [19] was obtained, with the additional assumption that the wind is in the positive $x$ direction (cf. Figure 1). The linear hydrodynamic sea model will be applied to our P-band $(0.3 \mathrm{GHz})$ and L-band $(1.2 \mathrm{GHz})$ simulations.

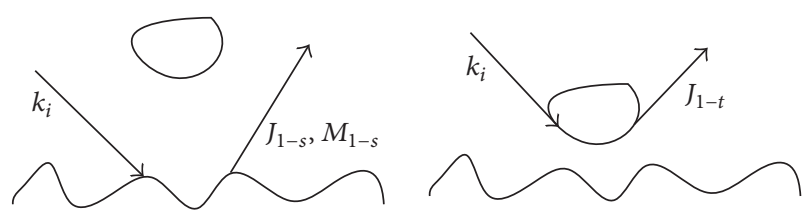

(a)

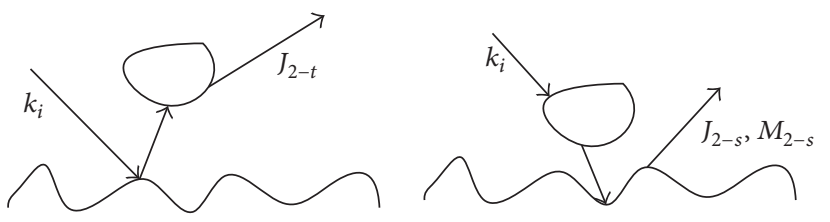

(b)
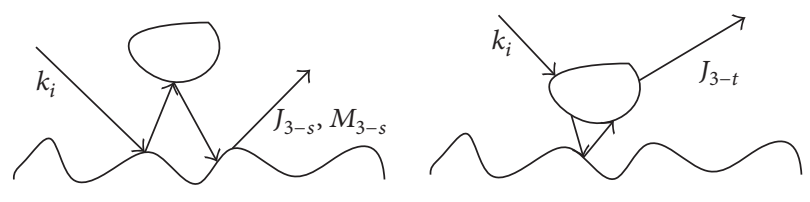

(c)

FIGURE 2: Multiple scattering models.

2.2. Theory of Hybrid PO-PO Method. In our previous work, the hybrid PO-PO method was utilized to calculate the EM scattering from a target above the sea surface; the method calculates the EM scattering from a target and a rough surface separately and then solves the mutual EM coupling between the target and the surface using an iterative process. The coupling process will not stop until it meets the condition of convergence; that is, when the induced currents on target and sea surface stabilize, iterations will stop.

Figure 2 illustrates the multiple scattering mechanisms of the composite model. The total scattering can be categorized into two types: The first is 1st-order (direct) scattering from sea surface when the target is assumed to be absent and 1storder scattering by target when the sea surface is assumed to be absent (cf. Figure 2(a)). $\mathbf{J}_{1-s}$ and $\mathbf{M}_{1-s}$ are the electric and magnetic currents induced by the incident wave on the dielectric sea surface. $\mathbf{J}_{1-t}$ represents the induced electric current on the PEC target surface. The subscripts $s$ and $t$ denote sea surface and target, and they are the same for subsequent variables. The second is mutual coupling scattering between target and sea surface as shown in Figures 2(b) and 2(c). In Figure 2(b), the incident wave arrives at the sea surface (target) initially and is scattered by its surface. Then, the scattered wave as incident wave travels from sea surface (target) to target (sea surface). Finally, the wave is scattered by target (sea surface). $\mathbf{J}_{2-s}, \mathbf{M}_{2-s}$, and $\mathbf{J}_{2-t}$ are the induced surface currents for the 2nd-order scattering. Similarly, the 3rd-order scattering is shown in Figure 2(c).

The key of the hybrid PO-PO method is the solution of the induced surface currents on target or sea surface for each scattered path. $\mathbf{J}_{n-s}, \mathbf{M}_{n-s}$, and $\mathbf{J}_{n-t}(n=1,2,3,4, \ldots)$ are solved by the PO theory and the equivalent principle. 
Moreover, the total surface currents on sea surface and target for multiple scattering can be written as

$$
\begin{aligned}
\mathbf{J}_{\text {total }-s} & =\mathbf{J}_{1-s}+\mathbf{J}_{2-s}+\mathbf{J}_{3-s}+\mathbf{J}_{4-s}+\cdots, \\
\mathbf{M}_{\text {total }-s} & =\mathbf{M}_{1-s}+\mathbf{M}_{2-s}+\mathbf{M}_{3-s}+\mathbf{M}_{4-s}+\cdots, \\
\mathbf{J}_{\text {total }-t} & =\mathbf{J}_{1-t}+\mathbf{J}_{2-t}+\mathbf{J}_{3-t}+\mathbf{J}_{4-t}+\cdots,
\end{aligned}
$$

Thus, the total scattered fields of composite model in the far zone can be easily obtained by the equivalent principle.

2.3. Reduction of Coupling Areas. The coupling process between the sea surface and target takes the majority of computing time by calculating the scattering of composite models. Numerical experiments showed that the scattering by rough surface is much stronger in the mirror direction than in other directions, and this property shows that the main scattering energy is concentrated in the specular direction, which is $10 \mathrm{~dB}$ higher than the scatterings in other directions [20]. Therefore, specular reflection property of the rough sea surface scattering is applied to reduce the coupling area on the sea surface.

$$
\begin{aligned}
& x_{1}=h \tan \theta_{i}-\frac{R}{\cos \theta_{i}}, \\
& x_{2}=h \tan \theta_{i}+\frac{R}{\cos \theta_{i}}, \\
& L_{0}=x_{2}-x_{1}=2 \cdot \frac{R}{\cos \theta_{i}} .
\end{aligned}
$$

Considering the specular reflection properties, Qi et al. [7] and Zhang and Sheng [20] presented their own coupling areas according to geometrical optical principle. We follow the coupling area proposed by Zhang and Sheng. In (3) and (4), the truncated coupling length $L_{0}$ is illustrated in Figure 1. $x_{1}$ and $x_{2}$ are the left and right boundaries of the truncated area, $R$ is the external diameter of the missile-like target, and $h$ is the height of the target.

In this paper, the scattering model is fit for the PO method. Once the surface electric and magnetic currents on the target and rough surface are determined, the scattered field can be calculated using the Huygens equivalence principle:

$$
\begin{aligned}
& \psi^{\text {sca }}\left(\theta_{s}, \mathbf{r} ; \theta_{i}\right)=\frac{\widehat{\mathbf{y}}}{4} \\
& \cdot \sqrt{\frac{2}{\pi k r}} e^{i k r} e^{-i(\pi / 4)} \int_{s}\left[(-i) J_{\text {total }}(\mathbf{r}) e^{-i \mathbf{r} \cdot \mathbf{k}_{s}}\right. \\
& \left.+k\left(\widehat{\mathbf{n}} \cdot \widehat{\mathbf{k}}_{s}\right) M_{\text {total }}(\mathbf{r}) e^{-i \mathbf{r} \cdot \mathbf{k}_{s}}\right] d s .
\end{aligned}
$$

In (5), $\psi^{\text {sca }}\left(\theta_{s}\right)$ is assumed to be the scattered field of the "farfield" region and can be applied to both target and rough sea surface. $J_{\text {total }}(\mathbf{r})$ and $M_{\text {total }}(\mathbf{r})$ are related to $\mathbf{J}_{\text {total }}$ and $\mathbf{M}_{\text {total }}$ on target or sea surface. $\mathbf{J}_{\text {total }}=-(\widehat{\mathbf{y}} / i \omega \mu) \cdot J_{\text {total }}(\mathbf{r}), \mathbf{M}_{\text {total }}=$ $(\widehat{\mathbf{y}} \times \widehat{\mathbf{n}}) \cdot M_{\text {total }}(\mathbf{r})$, and their specific form can be found in our previous study [13]. $\widehat{\mathbf{k}}_{s}$ is the scattering wave vector, and $k$ represents the electromagnetic wavenumber in free space. $\widehat{\mathbf{n}}$ is unit normal vector on the surface. For further analysis, introducing a normalized quantity called backscattering $\left(\theta_{s}=\right.$ $-\theta_{i}$ ) amplitude would be convenient [21].

$$
\begin{aligned}
& \gamma\left(\theta_{s}, \theta_{i}\right) \\
& \equiv \frac{\exp \left(-i k_{0} r\right) \sqrt{2 \pi r}\left[\psi^{\text {sca }}\left(\theta_{s}, \mathbf{r}_{t} ; \theta_{i}\right)+\psi^{\text {sca }}\left(\theta_{s}, \mathbf{r}_{s} ; \theta_{i}\right)\right]}{\left(\int\left|\psi^{i}(x, 0)\right|^{2} d x\right)^{1 / 2}}
\end{aligned}
$$

$\mathbf{r}_{t}$ and $\mathbf{r}_{s}$ represent the position vector of scattering source points on target and sea surface, respectively. $\psi^{i}(x, z)$ is a Thorsos tapered incident plane wave chosen to avoid artificial edge diffraction from the finite length rough surface $[22,23]$.

$$
\begin{aligned}
& \psi^{i}(x, z)=G(x, z) \cdot \exp \left[i k\left(x \sin \theta_{i}-z \cos \theta_{i}\right)\right], \\
& G(x, z)=\exp \left[\frac{i k\left(x \sin \theta_{i}-z \cos \theta_{i}\right)}{\left(k g \cos \theta_{i}\right)^{2}}\right. \\
& \left.\cdot\left(\frac{2\left(x+z \tan \theta_{i}\right)^{2}}{g^{2}}-1\right)-\frac{\left(x+z \tan \theta_{i}\right)^{2}}{g^{2}}\right] .
\end{aligned}
$$

\section{Results and Discussions}

All simulations were obtained on a computer with a $2.93 \mathrm{GHz}$ processor (Intel Core i3 CPU), 3.85 GHz memory, and Intel Visual Fortran compiler XE.

The validity of PO method for the scattering of sea surface should be considered. For the scattering of rough surface alone, the PO method is known as KA. For the conditions that meet $l_{c}>\lambda$ and $s=\sqrt{2} \sigma / l_{c}<0.25$, KA is applicable [20]. For the PM spectrum, both the root mean square (rms) height $\sigma$ and correlation length $l_{c}$ are related to wind speed $U$ [13]. Therefore, $s$ is the rms slope of the rough surface that is commonly used to describe the roughness of a surface.

According to numerical experiments by Zhang, accuracy of the final result is determined by the the coupling area $L_{0}$. Moreover, $L_{0}$ is related to the height of target $h$, target size $R$, and roughness of the underlying surface. In our simulations, $h$ is less than or equal to $5 \lambda, R$ is fixed to $2.5 \lambda$, and the wind speed is less than or equal to $7 \mathrm{~m} / \mathrm{s}$ (thus, $l_{c}>6 \lambda$ and $s<0.05$ meet the conditions of the PO described). The monostatic NRCS of the composite model in Figure 1 was calculated under these conditions, and we found that the choice of (4) derived rms error of $1.70 \mathrm{~dB}$ and $1.60 \mathrm{~dB}$ for the underlying PEC and dielectric sea surfaces, respectively. In this paper, $x_{1}$ and $x_{2}$ were extended by $3 h$ to the original left and right boundaries $\left(L_{0}=2\left(R / \cos \theta_{i}+3 h\right)\right)$, so that rms errors are $0.47 \mathrm{~dB}$ and $0.48 \mathrm{~dB}$, and the results are closer to the results of the entire coupling rough surface.

3.1. Validity and Efficiency of PO-PO Method. Numerical studies of the Doppler spectra are based on accurate and efficient electromagnetic (EM) backscattering simulations. In this paper, we used a sampling interval of $\lambda / 10$ [16] to generate a sea surface. To examine our scattering model, the monostatic NRCS of composite scattering from a PEC 
TABLE 1: Comparison of CPU time and memory.

\begin{tabular}{lccc}
\hline Model & Method & Time (s) & Memory (MB) \\
\hline \multirow{3}{*}{ Target + PEC sea surface } & PO-PO (total-coupling area) & 3706.6 & 3.2 \\
& PO-PO (local-coupling area) & 287.2 & 3.2 \\
\hline \multirow{2}{*}{ Target + dielectric sea surface } & MOM & 3289.9 & 304.0 \\
& PO-PO (total-coupling area) & 6306.1 & 3.5 \\
& PO-PO (local-coupling area) & 563.5 & 3.5 \\
\hline
\end{tabular}

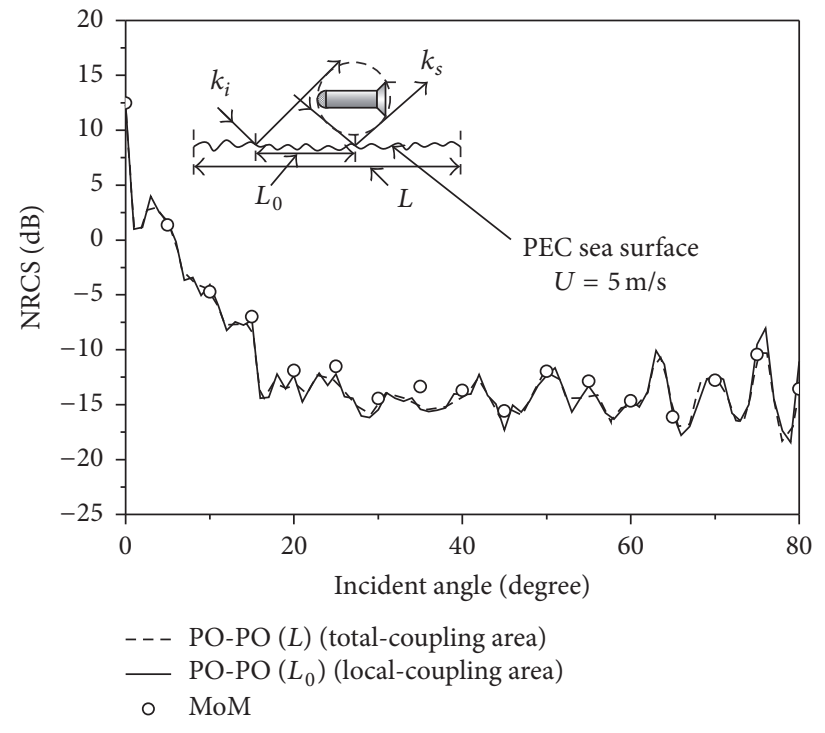

(a)

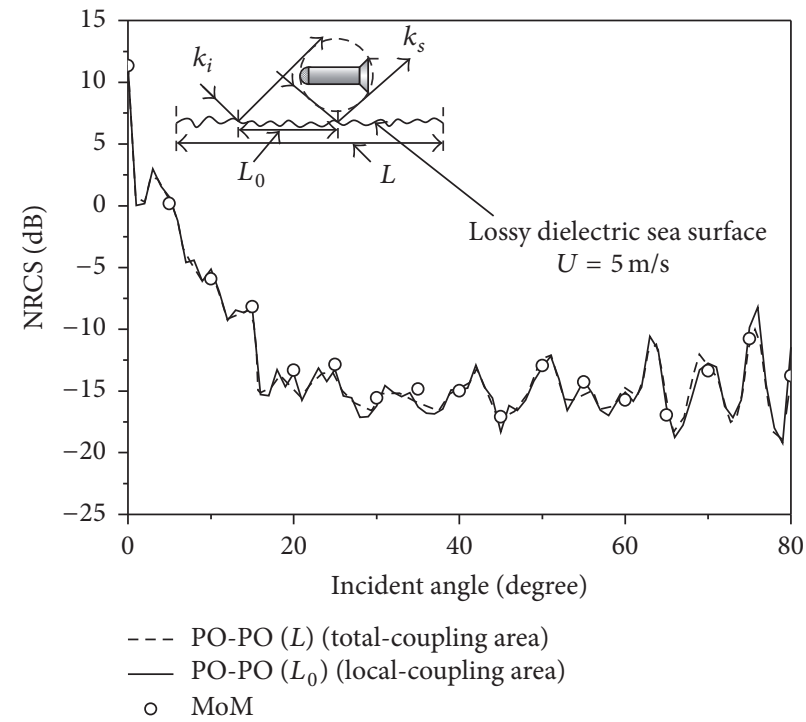

(b)

FIGURE 3: Comparison of NRCS by MOM and PO-PO: (a) PEC sea, (b) dielectric sea.

missile-like target above a $1 \mathrm{D}$ rough sea surface was calculated using the hybrid PO-PO method. The geometrical sizes of the target were assumed to be $4 \mathrm{~m}$ in length, $1 \mathrm{~m}$ in diameter, and with afterbody being $2 \mathrm{~m}$ (Figure 1). The result was compared with those obtained by the conventional MOM method [16].

Where an electromagnetic wavelength at P-band was used, the tapering parameter was $g=L / 4$; when we used $N=4096$ surface sampling with sea surface length thus being $L=409.6 \lambda$, the wind speed was $U=5 \mathrm{~m} / \mathrm{s}$. Most importantly, the rough sea surface length in our simulations met the conditions of tapered wave, because the incident angle was restricted to the angle of $80^{\circ}$ (the sea surface length is at least $L=331.6 \lambda$ ) [24]. The target had a height of $h=5 \mathrm{~m}$ and a boundary meshed into 350 segments. Based on the assumption that sea water temperature was $10^{\circ} \mathrm{C}$ and salinity was $30 \mathrm{ppt}$, the dielectric constant of sea water is $\varepsilon_{r}=(76.0,200.2)$ [25] in our P-band. For the hybrid POPO method, the total currents consisting of up to 4th-order scattering were calculated. The results calculated by the POPO method are shown according to different coupling areas, namely, total (entire sea surface area) and local (partial sea surface area controlled by (3)) areas. The numerical results were determined by averaging 20 Monte Carlo realizations.
As shown in Figure 3, the results by MOM and totalcoupling hybrid PO-PO match throughout all the observation angles for both $\mathrm{PEC}$ and actual dielectric rough sea surfaces. Moreover, the results from the local-coupling hybrid PO-PO are in good agreement with those from the conventional total-coupling hybrid PO-PO. It validates the efficiency of the local-coupling hybrid PO-PO method. Furthermore, the comparisons of computer resources consumed in memory and time for one composite realization from $0^{\circ}$ to $80^{\circ}$ by $\mathrm{PO}-\mathrm{PO}$ and MOM are also demonstrated in Table 1 . For the PEC rough sea surface case, the memory by local-coupling hybrid PO-PO was only $1.05 \%$ of that by MOM, and the calculation time consumed by local-coupling hybrid PO-PO was approximately $8.72 \%$ of that by MOM. For dielectric sea surface cases, our method is of great advantage, because the memory is only $0.31 \%$ of that by MOM, and the calculation time consumed is only $0.72 \%$ of that by MOM.

3.2. Doppler Analysis under Different Conditions. Doppler spectrum [8] is defined as

$$
S(f)=\frac{1}{T}\left|\int_{0}^{T} \gamma\left(t, \theta_{s}\right) \exp (i 2 \pi f t) d t\right|^{2} .
$$




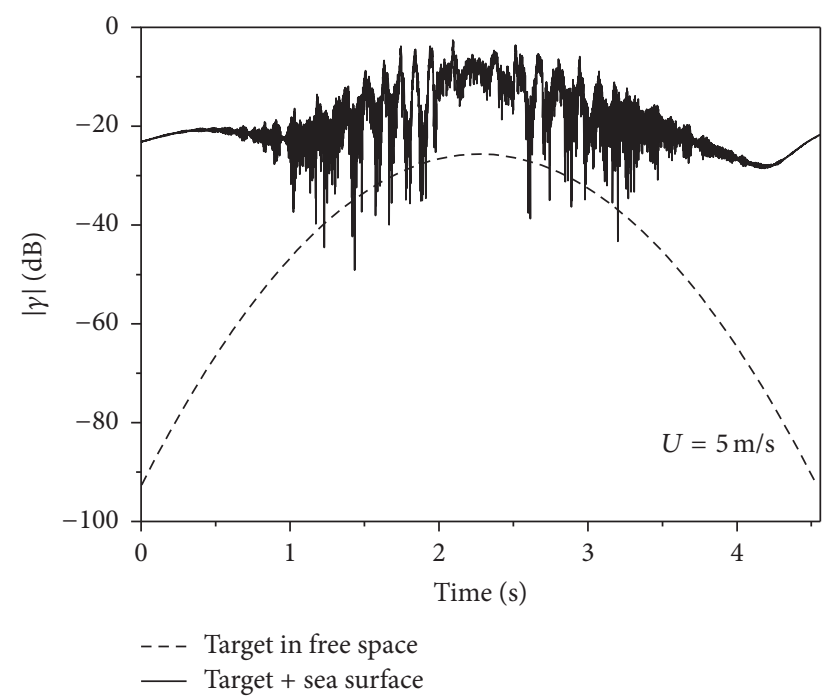

(a)

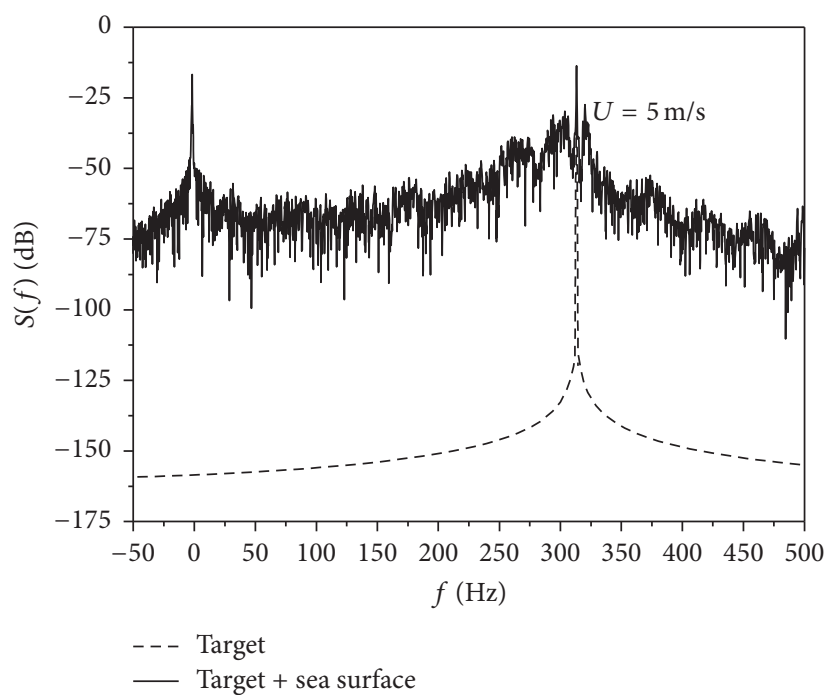

(b)

Figure 4: Target above sea surface and in free space. (a) Scattering amplitude. (b) Doppler spectra.

In the Doppler spectra simulations, the missile-like target above the sea surface was illuminated by the Thorsos tapered plane wave with tapering parameter $g=L / 6$. The geometrical sizes of the target are the same as in Figure 3, and the simulation sea surface length was $L=819.2 \mathrm{~m}$.

According to the theoretical analysis, the Bragg frequency of the sea clutters [9] and center Doppler frequency of a moving target with speed $V$ can be estimated.

$$
\begin{aligned}
& f_{B}\left(\theta_{i}\right)=\frac{1}{2 \pi} \sqrt{g \cdot 2 k_{0} \sin \theta_{i}}, \\
& f_{t}\left(\theta_{i}\right)=\frac{1}{2 \pi} \cdot 2 k_{0} V \sin \theta_{i} .
\end{aligned}
$$

First, we calculated the Doppler spectrum of the composite model at our P-band $(0.3 \mathrm{GHz})$. To further validate the results of the Doppler spectrum, we followed Liu and Jin [8]. The sea surface length was divided into 8192 surface samples, and the target was meshed into 350 segments. The missile-like target was flying at height $h=2 \mathrm{~m}$ with a speed of $V=600 \mathrm{~km} / \mathrm{h}$. For time-evolving simulations, the chosen step in the time domain was $0.001 \mathrm{~s}$. For each composite model realization, 4560 steps in the time domain were used, which meant that the simulation time lasted $4.56 \mathrm{~s}$. Based on the assumption that the target is flying in the negative $x$ direction (Figure 1) above the sea surface, the target was located at $380 \mathrm{~m}$ at $t=0 \mathrm{~s}$ and flies to $-380 \mathrm{~m}$ at $t=4.56 \mathrm{~s}$.

Figure 4 shows the scattering amplitude and Doppler spectra of the missile-like target flying in free space and above sea surface at the incident angle of $\theta_{i}=70^{\circ}$. The rough sea surface was driven by a wind speed of $U=5 \mathrm{~m} / \mathrm{s}$. In Figure 4(a), both amplitude modulation and phase modulation are on backscattering amplitude as the target flies above a PEC rough sea surface. In Figure 4(b), two Doppler peaks occur as the target flies above the PEC rough sea surface; these two peaks represent the movement of sea clutters and flying target, and the peaks occur at approximately $f_{B}=-1.75 \mathrm{~Hz}$

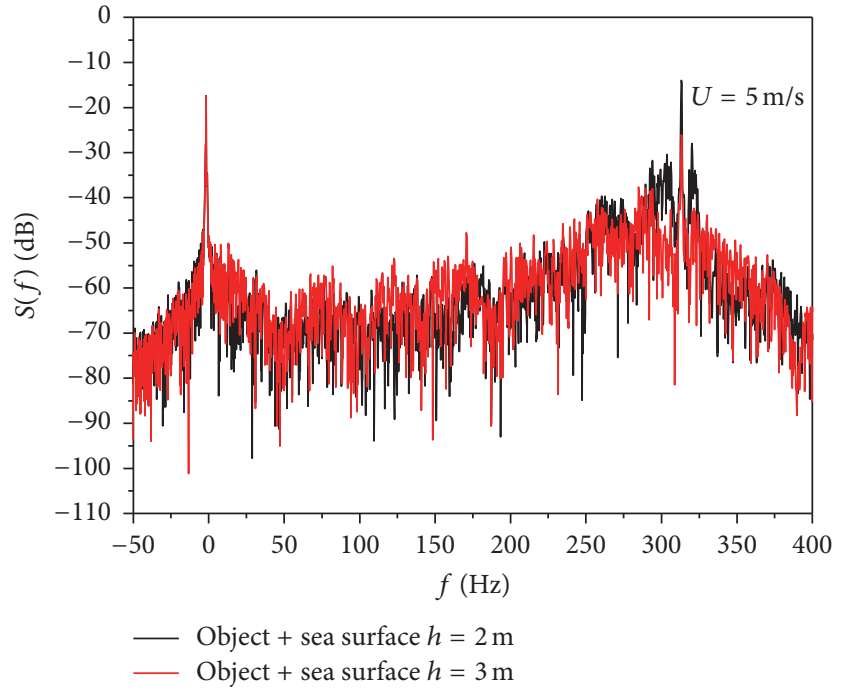

FIGURE 5: Doppler spectra at different heights.

and $f_{t}=313.16 \mathrm{~Hz}$, which are consistent with the analytical estimate given in (9). The result obtained by our method is the same as that obtained by Liu and Jin in tendency and conclusions.

Figure 5 compares the Doppler spectra from the target flying at heights of $2 \mathrm{~m}$ and $3 \mathrm{~m}$ above dielectric sea surface with a speed of $V=600 \mathrm{~km} / \mathrm{h}$. The incident angle was $70^{\circ}$, and the sea surface was driven by a speed of $U=5 \mathrm{~m} / \mathrm{s}$ with its dielectric constant still at $\varepsilon_{r}=(76.0,200.2)$. The main lobe of the target spectrum for the $3 \mathrm{~m}$ case decreased in amplitude and shrank in width. The phenomena can be explained by the image principle and two-source array radiation [26], and the phase difference between the target and its image minimizes coherent backscattered returns at $h=2.92 \mathrm{~m}$ theoretically in our simulation. In addition, the image source affects the 


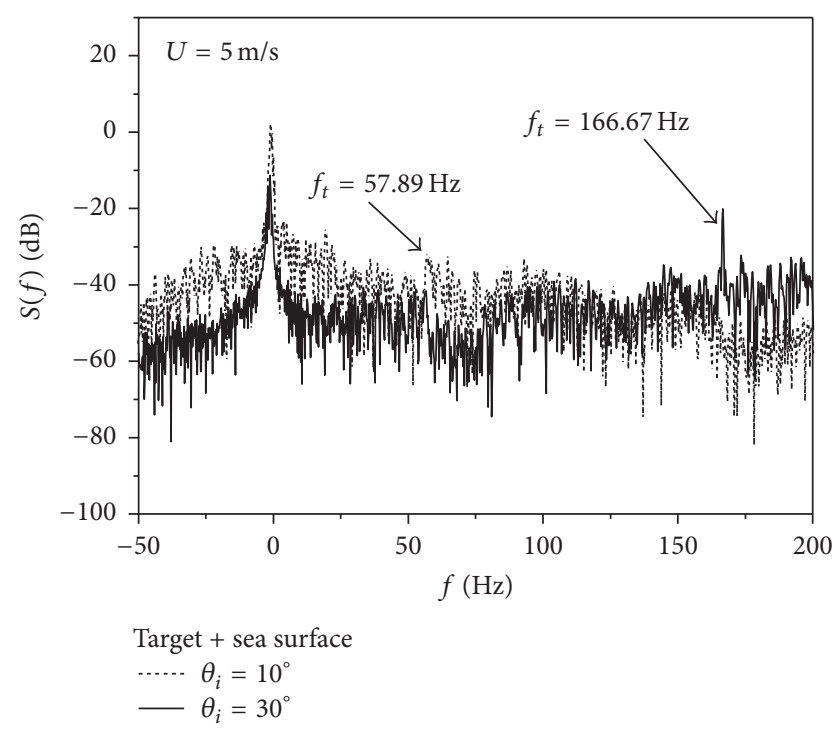

FIgURE 6: Doppler spectra from different incidence angles.

coherent backscattering periodically with the increase of target altitudes, and the target may not be detected at some specific altitudes.

Figure 6 shows the Doppler spectra from the target flying above the dielectric sea surface from different incident angles of $10^{\circ}$ and $30^{\circ}$. The target height is $2 \mathrm{~m}$, and other target and sea surface parameters are the same as those in Figure 5. As the incident angle increased, the sea clutter spectrum decreased in amplitude, and the main lobe of the target spectrum became clearer and easier to distinguish. The primary reason is that the influence of backscattering from the sea surface was large with respect to that from the composite model for the small incident angle, and the Doppler frequency shift of the target was smaller than that of the total composite backscattering; thus, the main lobe was totally merged into the clutters for the incident angle of $10^{\circ}$. With the increased incident angle, the amplitude of backscattering from the sea surface will become decreased; moreover, when the Doppler frequency shift of the target is large, the target emerges in the Doppler spectrum of the backscattering from the sea surface.

Figure 7 presents the Doppler spectra from the target flying above sea surfaces when driven by different wind speeds at an altitude of $2 \mathrm{~m}$. Other parameters are the same as those in Figure 5. For a clear comparison, the main spectral lobes of sea clutters and flying target were plotted. Figure 7 shows that the Doppler spectra of sea clutters and flying target both broadened, but the amplitude became higher for case of the sea clutters and lower for the target case as wind speed increased. This condition is mainly due to the incoherent scattering that increases as the roughness of the sea surface increases. Thus, the backscattering energy is distributed over a wide region in the frequency domain that has been discussed in our previous works [27].

The effect of the speed of flying target with an altitude of $2 \mathrm{~m}$ on the Doppler of the composite model is illustrated in Figure 8 . Sea surface and incident wave parameters are the same as those shown in Figure 5. For different speeds of flying target, the Doppler spectra of sea clutters are coincidental. Moreover, different speeds of flying target had almost no effect on the Doppler spectra of sea clutters. If we move one of the two target spectrum peaks to the other, the two target spectrum peaks and their side lobes coincide. The peaks of the target spectrum only appear at the positions where they are decided by (9).

Then, the coupling effects on Doppler spectra of the composite model are discussed, the target height was $2 \mathrm{~m}$ with speed of $V=600 \mathrm{~km} / \mathrm{h}$ and wind speed of $5 \mathrm{~m} / \mathrm{s}$, and the underlying sea surface was dielectric. For the results obtained in our L-band $(1.2 \mathrm{GHz})$, the chosen step in the time domain was $0.00038 \mathrm{~s}$. For convenience, we still simulated the model in the same time period $T=4.56 \mathrm{~s}$; thus, 12000 steps were performed in the time domain. The sea surface length was divided into 32768 surface samples, and the target was meshed into 750 segments. The dielectric constant of sea water was $(75.5,56.0)[25]$ in our L-band.

3.3. Coupling Effects on Doppler Spectra. To explore the effects of multiple scattering field of the PO-PO method on the spectra of composite model, Doppler spectra of each order scattering filed are calculated. Figure 9 shows the Doppler spectra of multiple scattering fields with different incident angles in our P-band. To be clear, the high-order Doppler spectra of target and sea surface were related to the coupling effects between the missile-like target and timevarying sea surface. Most notably, Figure 9 can be regarded as a detailed component of Figure 6. Intuitively, we can find that the 1st-order scattering from the target and sea surface was dominant, and as the order number of mutual coupling scatterings increased, the contribution of target and sea surface scattering to the total Doppler spectra decreased. Moreover, broadening of the Doppler spectra became clearer.

In Figure 9(b), the distributions of the Doppler spectra of the composite scattering model are almost similar to those from the 1st-order scattering from the sea surface, and the 1st-order Doppler spectra from the target are small in magnitude (as shown in Figure 9(a)). In addition, the high-order Doppler spectra from target and sea surface concentrated on the side of the Doppler spectra of of sea; therefore, the target spectra were entirely covered by the spectra of the sea clutters. However, as the incident angle increased, the magnitude of the 1st-order spectra of the target reached the same level as that of the 1st-order spectra of the sea surface (Figure 9(c)). The high-order Doppler spectra from the target and sea surface concentrated on the side of the Doppler spectra of target; therefore, the main spectrum lobe of the target appeared out of the spectrum of the sea clutter. Through our simulations, we found that, with the further increase of incident angle, the contribution of mutual coupling scattering to the total Doppler spectra increased, and even the 2ndorder scattering became dominant to the total Doppler spectrum at low grazing angles. The reason for the aforementioned phenomenon is explained in Figure 5. According to the results of our simulations, the width of the main Doppler spectrum lobe for the composite backscattering 


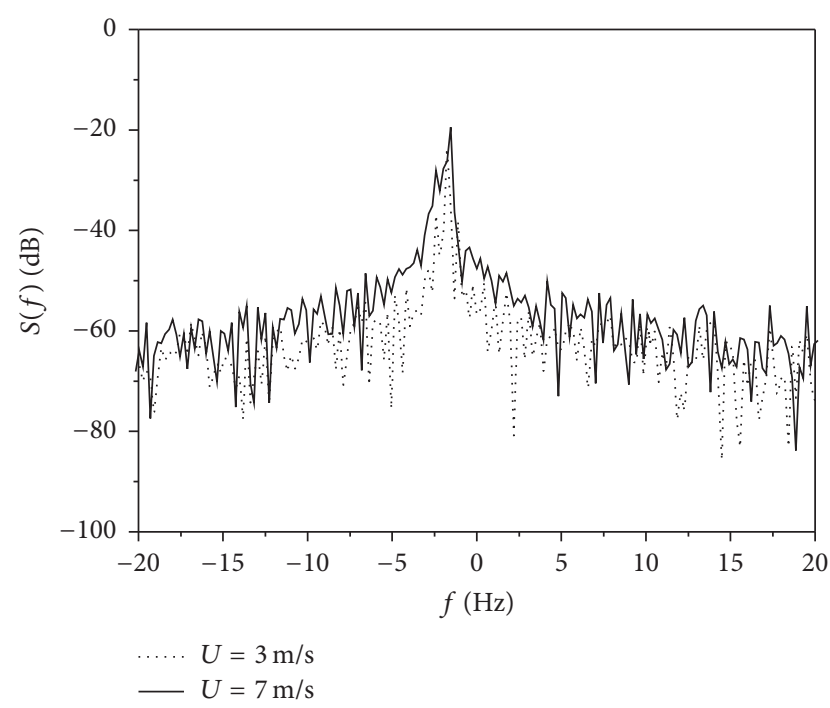

(a)

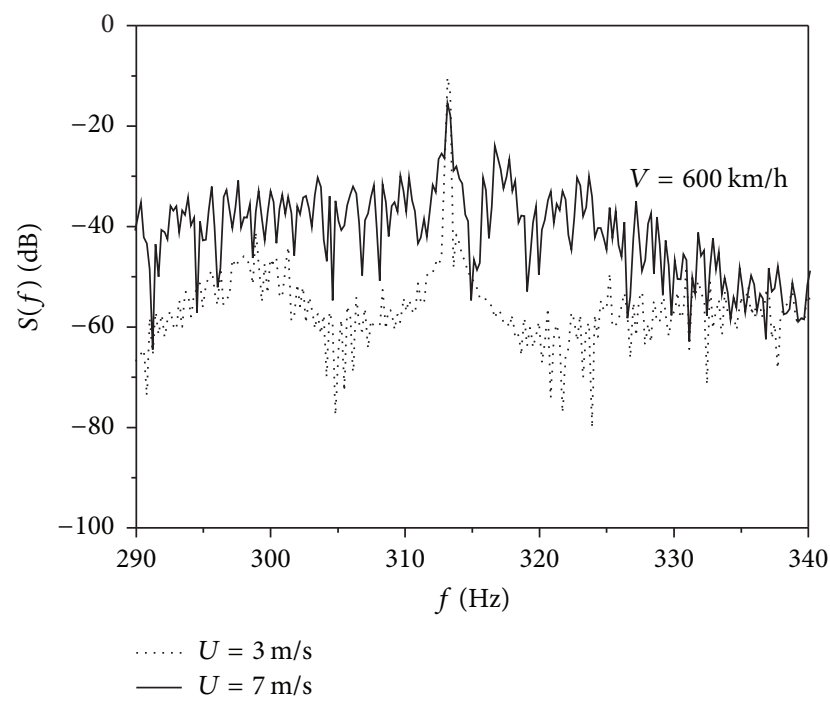

(b)

Figure 7: Doppler spectra at different wind speeds: (a) sea clutters, (b) target.

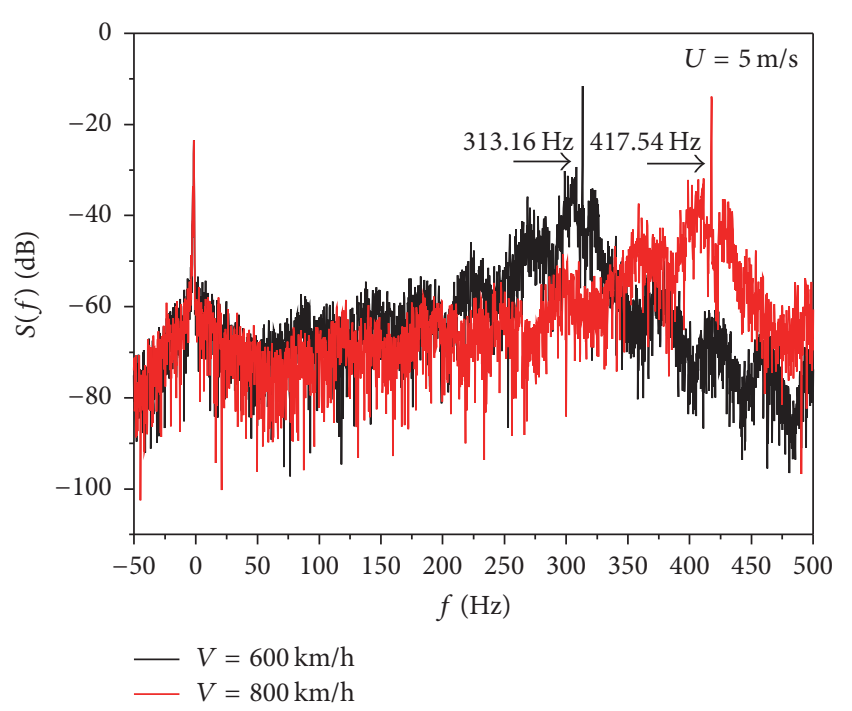

FIGURE 8: Doppler spectra of the target flying at different speed.

and sea surface decreased as the incident angle increased, which is consistent with theoretical prediction [28].

To further explore the coupling effects on Doppler spectra of the composite model, the 1st-order scatterings of target and sea surface were combined and compared with the total scattering of the composite model. The incident angle was $30^{\circ}$, and Figure 10 shows that if the mutual coupling scattering is considered between the sea surface and the missile-like target, an evident broadening of the Doppler spectrum is observed. Two main peaks are centered at the theoretical Doppler frequencies corresponding to the evolution of the sea and the velocity of the target. The coupling effects on the target spectrum were more evident than those on the sea clutter spectrum. The EM scattering from the sea surface significantly affected the backscattering
TABLE 2: Comparisons of CPU time of different types of underlying sea surface at our P-band.

\begin{tabular}{lccc}
\hline Sea type & Band & Time & $\times 4560$ \\
\hline PEC sea surface & $\mathrm{P}$ & $2.4 \mathrm{~s}$ & $3.04 \mathrm{hr}$ \\
Dielectric sea surface & $\mathrm{P}$ & $4.9 \mathrm{~s}$ & $6.21 \mathrm{hr}$ \\
\hline
\end{tabular}

TABLE 3: Comparisons of CPU time of different types of underlying sea surface at our L-band.

\begin{tabular}{lccc}
\hline Sea type & Band & Time & $\times 12000$ \\
\hline PEC sea surface & $\mathrm{L}$ & $9.8 \mathrm{~s}$ & $32.7 \mathrm{hr}$ \\
Dielectric sea surface & $\mathrm{L}$ & $20.1 \mathrm{~s}$ & $67.0 \mathrm{hr}$ \\
\hline
\end{tabular}

of the target. However, the target had minimal influence on the sea surface scattering. Additionally, we found that as the incident frequency increased, the influence of coupling field on the sea surface increased as well. Therefore, the mutual coupling between the sea surface and the target makes the transmitting paths of the EM wave much more complex. Thus, more spurious scattering can be found in the Doppler spectra. At certain frequencies, several spikes were found in the Doppler spectra. However, compared with the Bragg peak, these spikes were lower.

3.4. Computation Time of Doppler Spectra. Doppler spectra from different target heights, different wind speeds, and different target speeds at our L-band are not listed in this paper because their conclusions are the same as those of the P-band. In our simulations, the actual dielectric rough sea surface can be treated as a perfect conducting surface for Pand L-band simulations, and the result was accurate enough.

Comparisons of time consumed between different types of underlying sea surface are listed in Tables 2 and 3. Table 2 is for the case of our P-band, and Table 3 is for the case of 


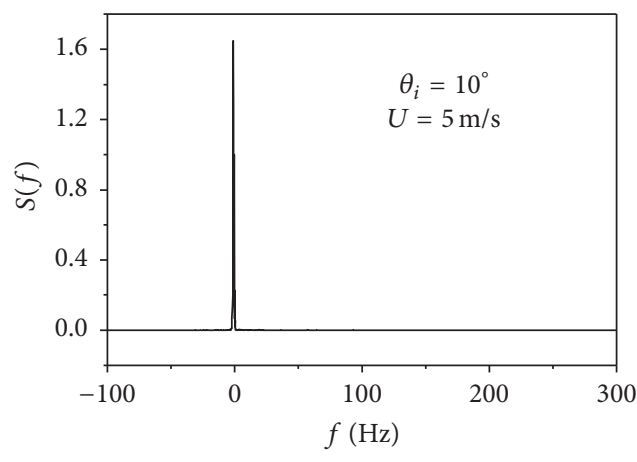

_ Total scattering

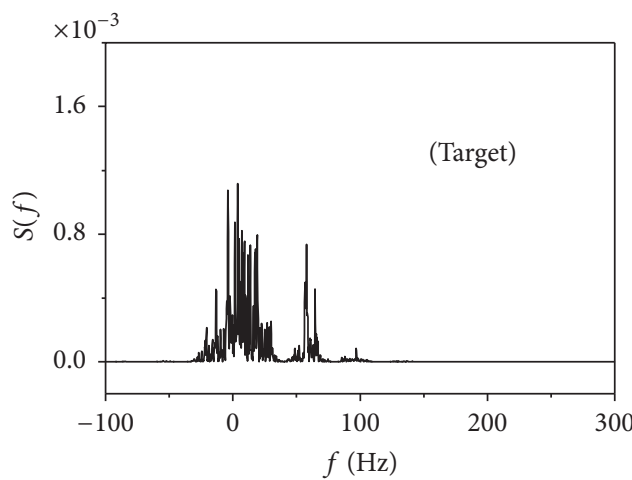

— 2nd-order scattering

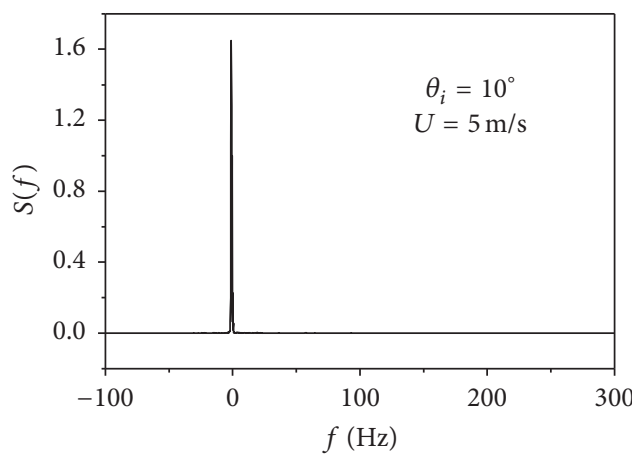

_ Total scattering

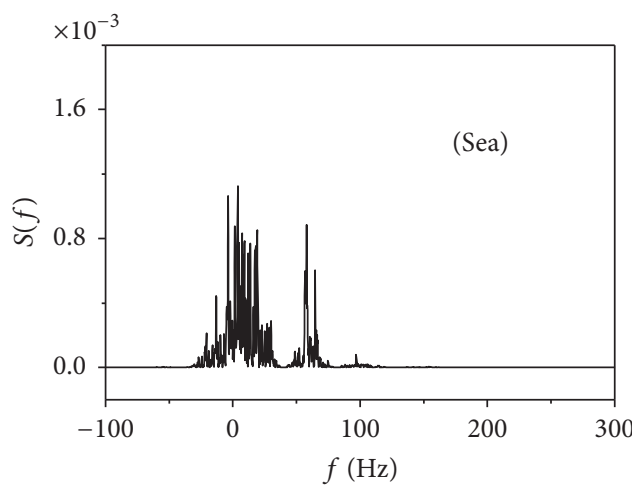

__ 2nd-order scattering

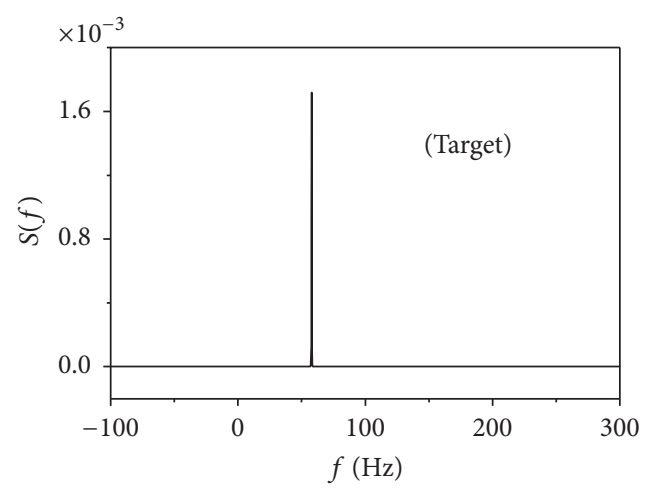

_ 1st-order scattering

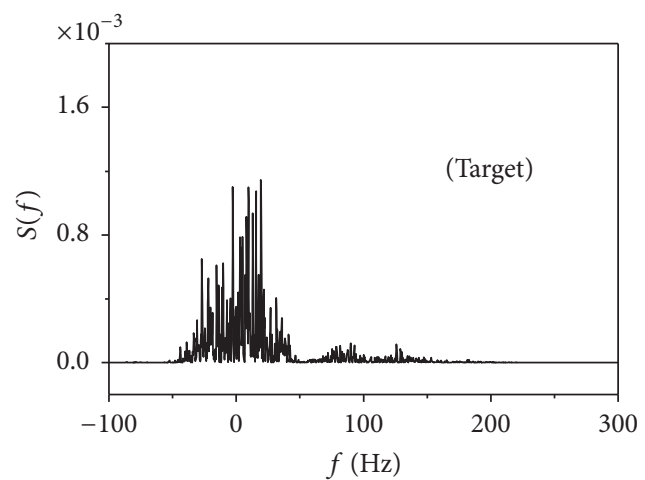

__ 4th-order scattering

(a)

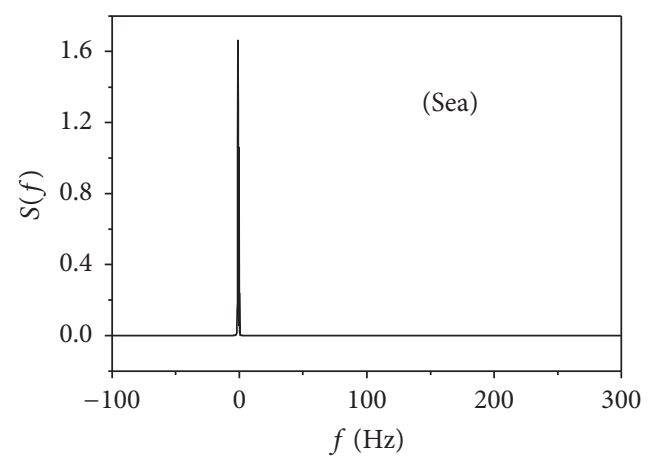

— 1st-order scattering

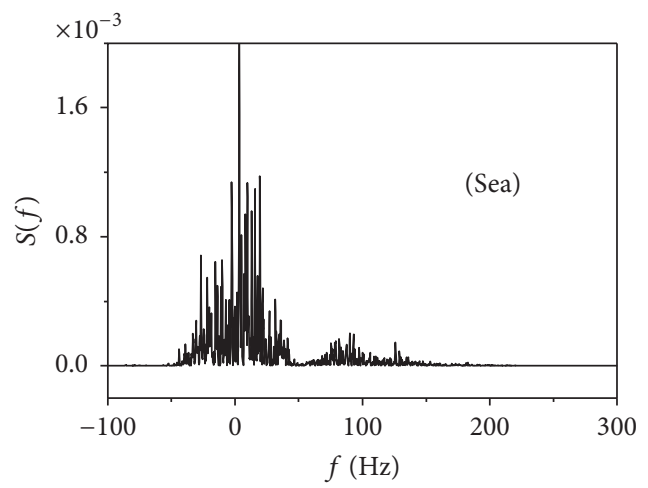

4th-order scattering

(b)

Figure 9: Continued. 

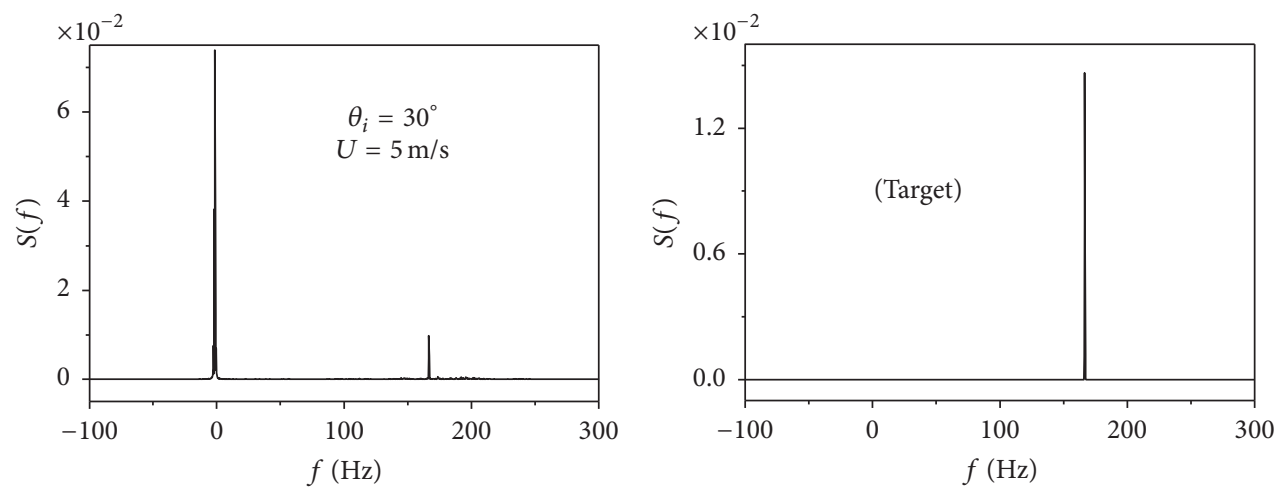

- Total scattering

_ 1st-order scattering
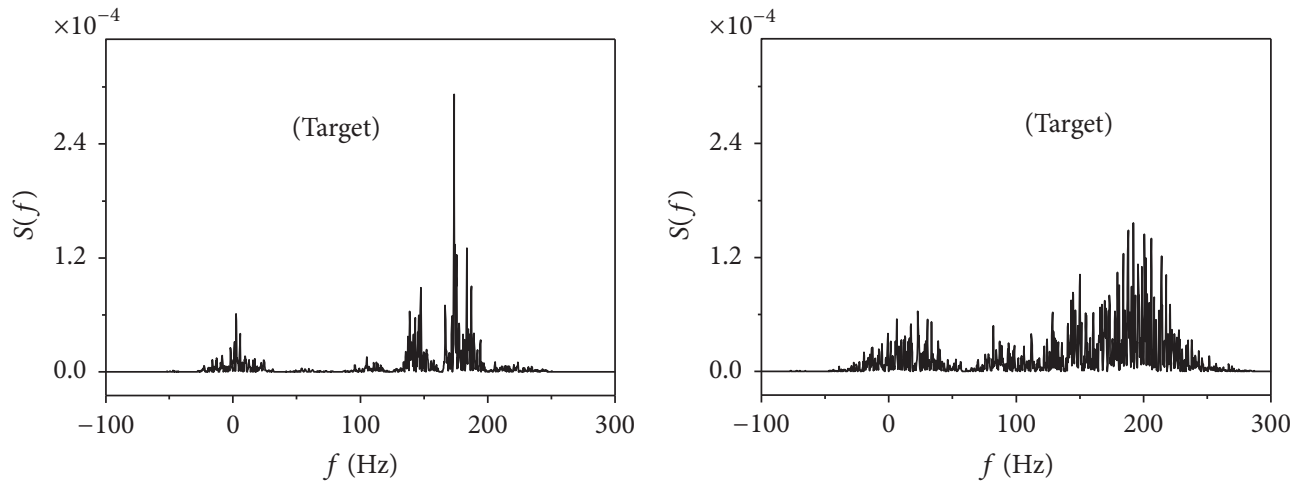

_ 2nd-order scattering

— 4th-order scattering

(c)
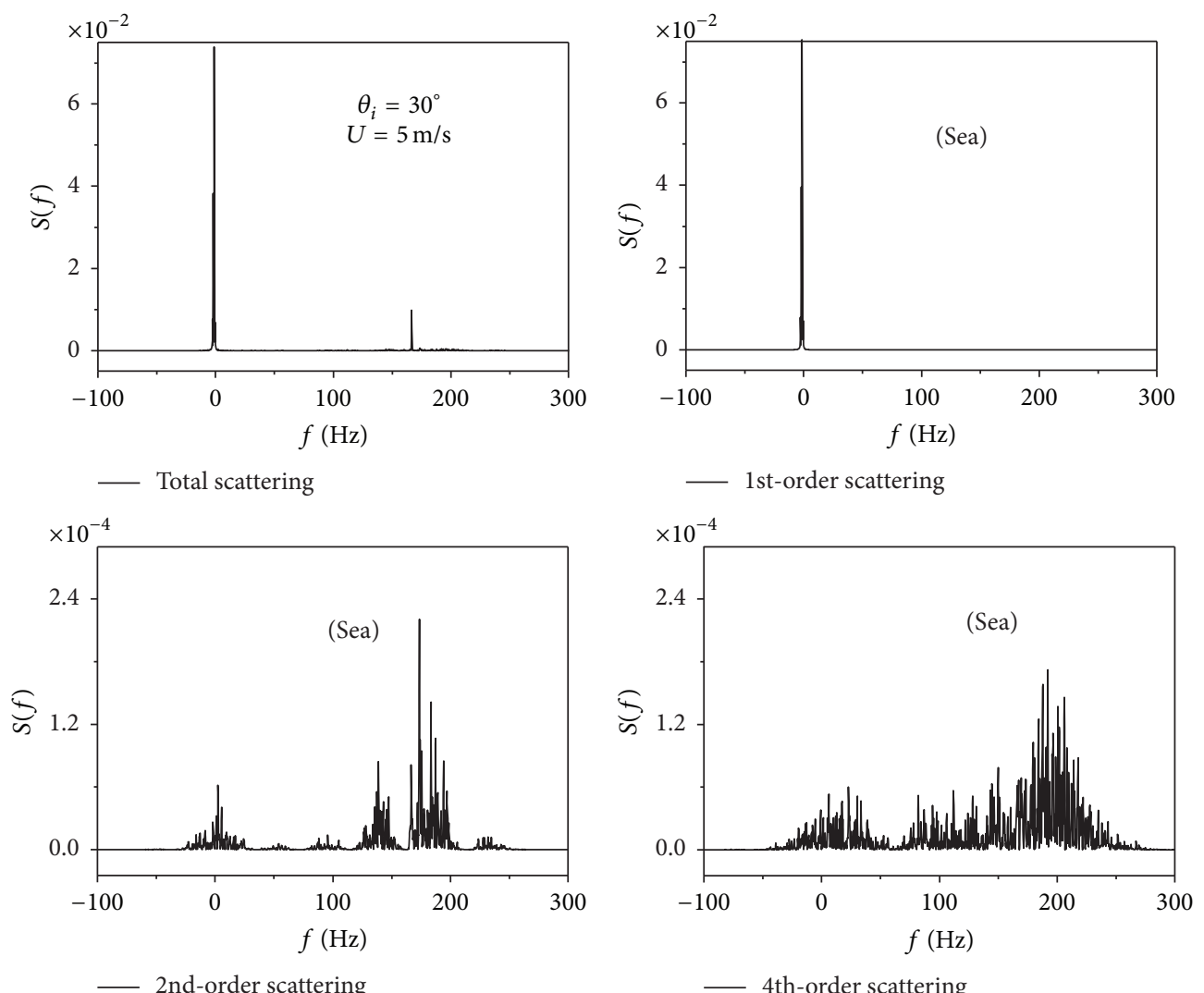

(d)

Figure 9: Doppler spectra of multiple scattering field. (a) Target $\left(\theta_{i}=10^{\circ}\right)$. (b) Sea surface $\left(\theta_{i}=10^{\circ}\right)$. (c) Target $\left(\theta_{i}=30^{\circ}\right)$. (d) Sea surface $\left(\theta_{i}\right.$ $=30^{\circ}$ ). 


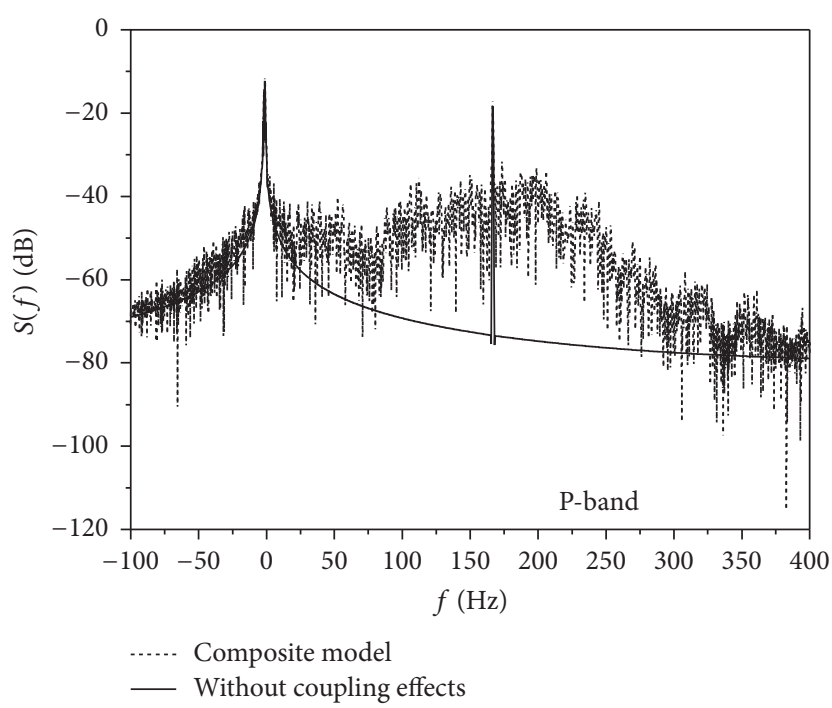

(a)

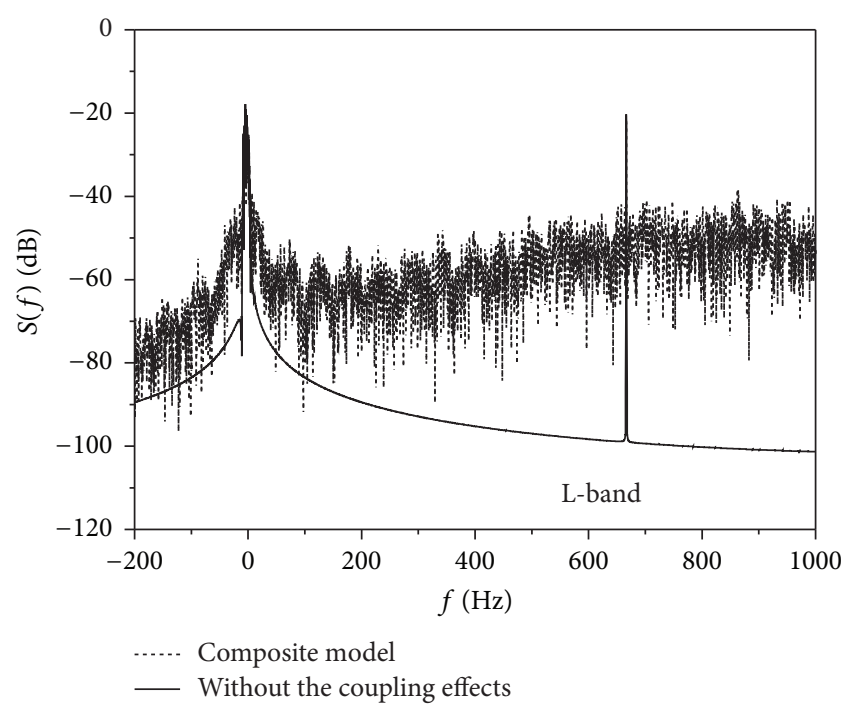

(b)

FIgURE 10: The coupling effects on the Doppler spectrum of the composite model. (a) P-band, (b) L-band.

our L-band. During the simulation of scattering amplitude, calculating the total currents on target and sea surface by using the PO-PO method was the most time-consuming procedure. The time consumed by the procedure of mesh generating, preprocessing, and far-field calculation was negligible. We found that our method saves times compared with the method proposed by Liu and Jin [8].

\section{Conclusions}

In this paper, a numerical electromagnetic method based on $\mathrm{PO}-\mathrm{PO}$ was used to calculate Doppler spectra from a missilelike target above the sea surface. The results were in good agreement with those obtained by the conventional MOM. Furthermore, based on the specular reflection properties of the rough surface, a reduction principle of the rough sea surface was used. The runtime showed that the hybrid POPO method enabled large-scale time-varying Monte Carlo simulations.

Doppler spectra of backscattered signals from the model were discussed in detail for different incident angles, frequencies, target altitudes, wind speeds, and target speeds at the incidence of horizontal polarization wave. The effects of multiple scattering field of the PO-PO method on the spectra of the composite model were discussed. We also found that if the mutual coupling scattering was considered between the sea surface and the missile-like target, the Doppler spectrum would broaden. Finally, the CPU times of different types of underlying sea surface at our P- and L-bands were compared. Treating the sea as PEC surface at our P- and L-bands can save time.

Our study was limited to 2D targets, horizontally polarized incidence, linear sea surface, and 1D sea surface model. With further improvement of computation resources, the simulation can be extended to 3D composite models, higher frequencies, vertically polarized incidence, and lossy nonlinear sea surface model.

\section{Competing Interests}

The authors declare that there is no conflict of interests regarding the publication of this paper.

\section{Acknowledgments}

This work was supported by the National Natural Science Foundation for Distinguished Young Scholars of China (Grant no. 61225002), the National Natural Science Foundation of China (Grant no. 61501360), and the Fundamental Research Funds for the Central Universities (Grant no. K5051007001).

\section{References}

[1] S. Martin, An Introduction to Ocean Remote Sensing, Cambridge University Press, New York, NY, USA, 2014.

[2] F. Jangal, S. Saillant, and M. Hélier, "Wavelet contribution to remote sensing of the sea and target detection for a highfrequency surface wave radar," IEEE Geoscience and Remote Sensing Letters, vol. 5, no. 3, pp. 552-556, 2008.

[3] R. Romeiser and D. R. Thompson, "Numerical study on the along-track interferometric radar imaging mechanism of oceanic surface currents," IEEE Transactions on Geoscience and Remote Sensing, vol. 38, no. 1, pp. 446-458, 2000.

[4] Y. Wang and Y. Zhang, "Investigation on Doppler shift and bandwidth of backscattered echoes from a composite sea surface," IEEE Transactions on Geoscience and Remote Sensing, vol. 49, no. 3, pp. 1071-1081, 2011.

[5] P. Liu and Y.-Q. Jin, "The finite-element method with domain decomposition for electromagnetic bistatic scattering from the 
comprehensive model of a ship on and a target above a largescale rough sea surface," IEEE Transactions on Geoscience and Remote Sensing, vol. 42, no. 5, pp. 950-956, 2004.

[6] L. Guo, Y. Liang, and Z. Wu, "A study of electromagnetic scattering from conducting targets above and below the dielectric rough surface," Optics Express, vol. 19, no. 7, pp. 5785-5801, 2011.

[7] C. Qi, Z. Zhao, and Z.-P. Nie, "Numerical approach on Doppler spectrum analysis for moving targets above a time-evolving sea surface," Progress in Electromagnetics Research, vol. 138, pp. 351365, 2013.

[8] P. Liu and Y.-Q. Jin, "Numerical simulation of the doppler spectrum of a flying target above dynamic oceanic surface by using the FEM-DDM method," IEEE Transactions on Antennas and Propagation, vol. 53, no. 2, pp. 825-832, 2005.

[9] J. V. Toporkov and G. S. Brown, "Numerical simulations of scattering from time-varying, randomly rough surfaces," IEEE Transactions on Geoscience and Remote Sensing, vol. 38, no. 4, pp. 1616-1625, 2000.

[10] J. T. Johnson, "A numerical study of scattering from an object above a rough surface," IEEE Transactions on Antennas and Propagation, vol. 50, no. 10, pp. 1361-1367, 2002.

[11] M. Djordjevic and B. M. Notaros, "Enhanced higher order MoM-PO modeling using multiple reflections in the $\mathrm{PO}$ region," in Proceedings of the IEEE Antennas and Propagation Society International Symposium, pp. 2905-2908, Albuquerque, NM, USA, July 2006.

[12] J. Li, L. Guo, and S. Chai, "Composite electromagnetic scattering from an object situated above rough surface," Applied Optics, vol. 53, no. 35, pp. 8189-8196, 2014.

[13] J. Li, L.-X. Guo, S.-R. Chai, and Y.-C. Jiao, "Electromagnetic scattering from a PEC object above a dielectric rough sea surface by a hybrid PO-PO method," Waves in Random and Complex Media, vol. 25, no. 1, pp. 60-74, 2015.

[14] S.-R. Chai, L.-X. Guo, and R. Wang, "PO-PO method for electromagnetic backscattering from a $2 \mathrm{D}$ arbitrary dielectriccoated conducting target located above a $1 \mathrm{D}$ randomly rough surface: horizontal polarisation," IET Microwaves, Antennas \& Propagation, vol. 8, no. 15, pp. 1340-1347, 2014.

[15] K. Li, L. Guo, J. Li, and S. Chai, "Composite EM scattering and Doppler spectral analysis for time-varying Sea surfaces and a target above it," in Proceedings of the Asia-Pacific Microwave Conference (APMC '15), pp. 1-3, Nanjing, China, December 2015.

[16] L. Tsang, J. A. Kong, K.-H. Ding, and C. O. Ao, Scattering of Electromagnetic Waves, Numerical Simulations, John Wiley \& Sons, 2004.

[17] C. L. Rino, T. L. Crystal, A. K. Koide, H. D. Ngo, and H. Guthart, "Numerical simulation of backscatter from linear and nonlinear ocean surface realizations," Radio Science, vol. 26, no. 1, pp. 5171, 1991.

[18] W. J. Pierson Jr. and L. Moskowitz, "A proposed spectral form for fully developed wind seas based on the similarity theory of SA Kitaigorodskii," DTIC Document, 1963.

[19] R. Wang and L.-X. Guo, "Study on electromagnetic scattering from the time-varying lossy dielectric ocean and a moving conducting plate above it," Journal of the Optical Society of America A, vol. 26, no. 3, pp. 517-529, 2009.

[20] X.-Y. Zhang and X.-Q. Sheng, "Highly efficient hybrid method for monostatic scattering by objects on a rough surface," IET Microwaves, Antennas \& Propagation, vol. 4, no. 10, pp. 15971604, 2010.
[21] J. V. Toporkov and G. S. Brown, "Numerical study of the extended Kirchhoff approach and the lowest order small slope approximation for scattering from ocean-like surfaces: Doppler analysis," IEEE Transactions on Antennas and Propagation, vol. 50, no. 4, pp. 417-425, 2002.

[22] E. I. Thorsos, "The validity of the Kirchhoff approximation for rough surface scattering using a Gaussian roughness spectrum," The Journal of the Acoustical Society of America, vol. 83, no. 1, p. 78, 1988.

[23] J. V. Toporkov, R. I. S. Awadallah, and G. S. Brown, "Issues related to the use of a Gaussian-like incident field for lowgrazing-angle scattering," Journal of the Optical Society of America A, vol. 16, no. 1, pp. 176-187, 1999.

[24] H. Ye and Y.-Q. Jin, "Parameterization of the tapered incident wave for numerical simulation of electromagnetic scattering from rough surface," IEEE Transactions on Antennas and Propagation, vol. 53, no. 3, pp. 1234-1237, 2005.

[25] T. Meissner and F. J. Wentz, "The complex dielectric constant of pure and sea water from microwave satellite observations," IEEE Transactions on Geoscience and Remote Sensing, vol. 42, no. 9, pp. 1836-1849, 2004.

[26] P. Liu and Y.-Q. Jin, "Numerical simulation of bistatic scattering from a target at low altitude above rough sea surface under an EM-wave incidence at low grazing angle by using the finite element method," IEEE Transactions on Antennas and Propagation, vol. 52, no. 5, pp. 1205-1210, 2004.

[27] L. Guo, Y. Wang, and Z. Wu, "Study on electromagnetic backscattering and Doppler spectrum of a moving spherical target above time-varying sea surface," Science in China, Series G: Physics, Mechanics and Astronomy, vol. 51, no. 3, pp. 269-281, 2008.

[28] F. G. Bass, I. M. Fuks, A. I. Kalmykov, I. E. Ostrovsky, and A. D. Rosenberg, "Very high frequency radiowave scattering by a disturbed sea surface part II: scattering from an actual sea surface," IEEE Transactions on Antennas and Propagation, vol. 16, no. 5 , pp. 560-568, 1968. 


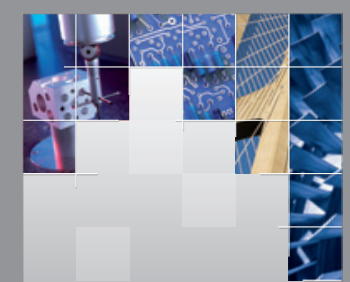

\section{Enfincering}
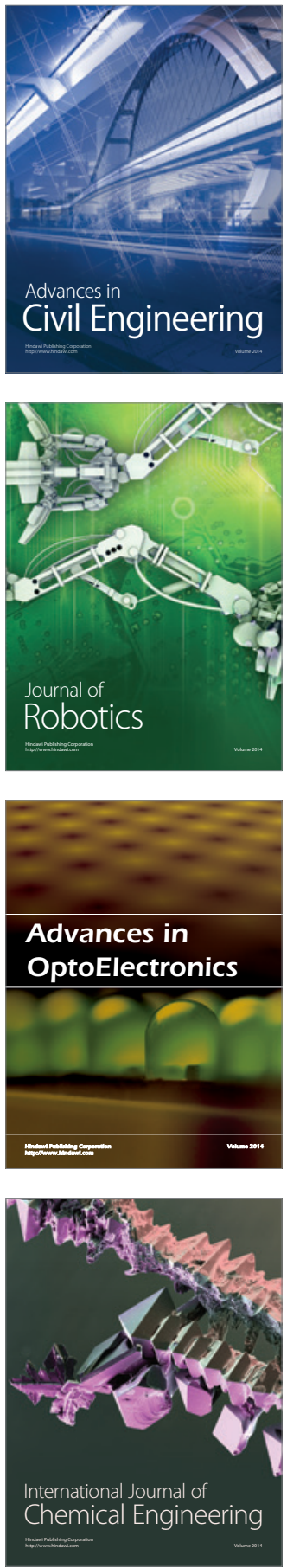

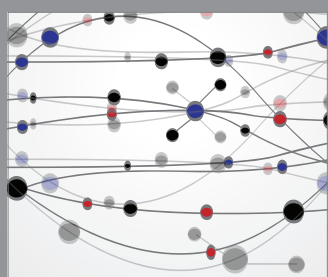

The Scientific World Journal

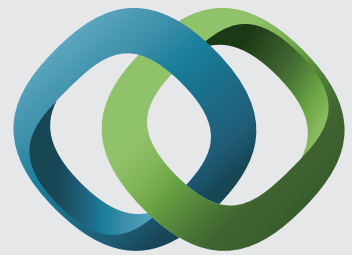

\section{Hindawi}

Submit your manuscripts at

http://www.hindawi.com
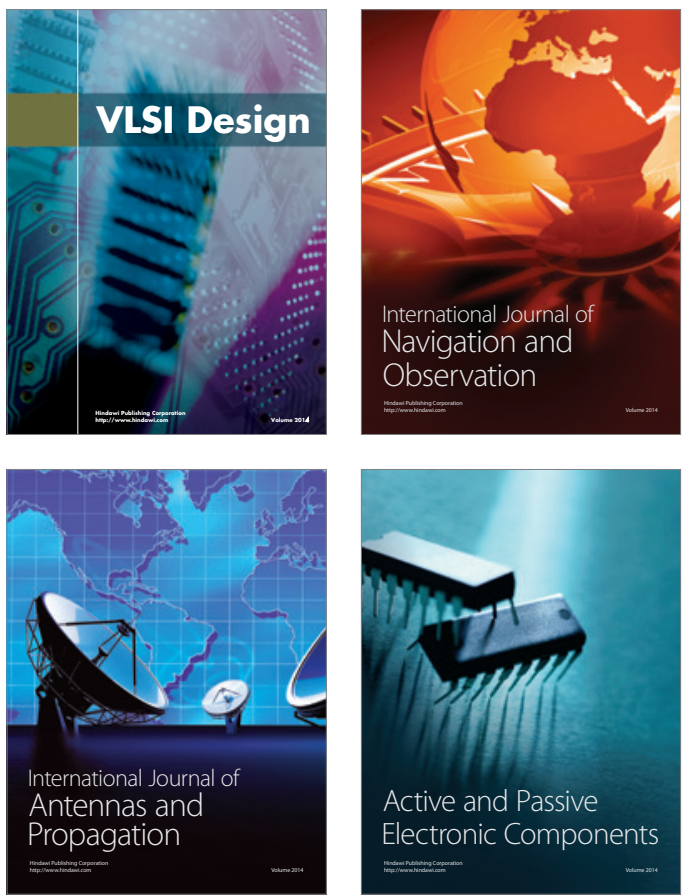
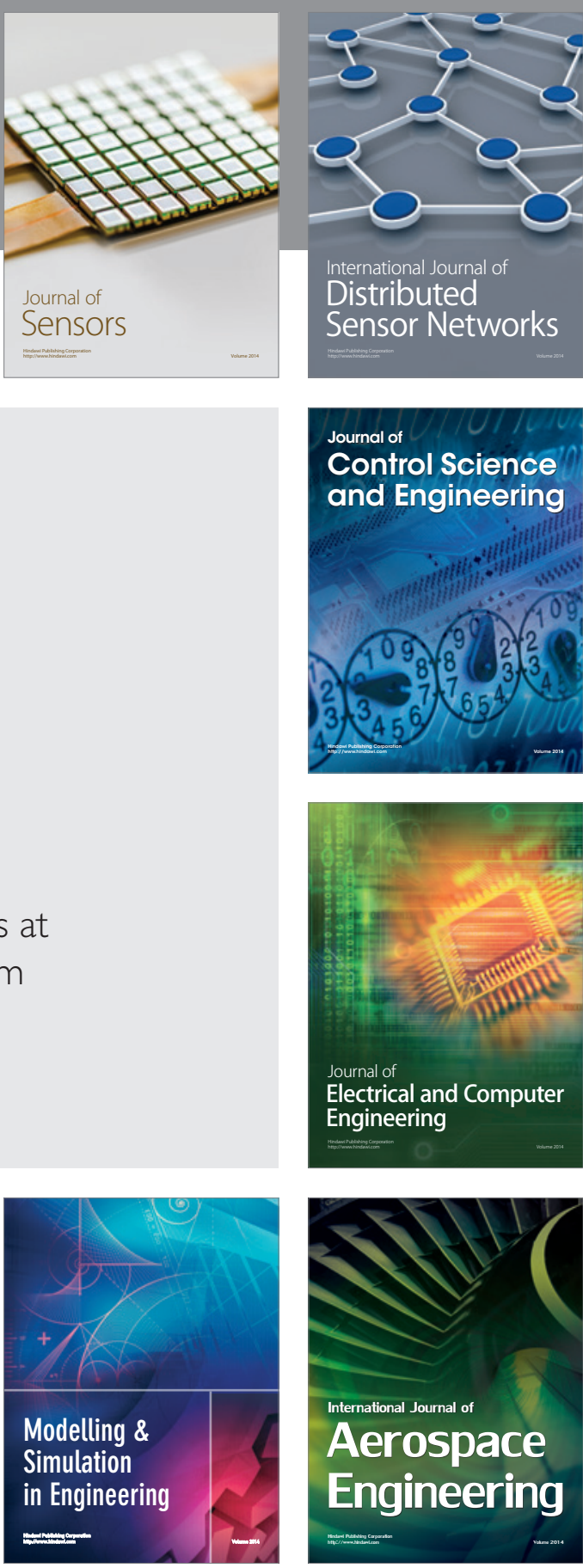

International Journal of

Distributed

Sensor Networks

Journal of

Control Science

and Engineering
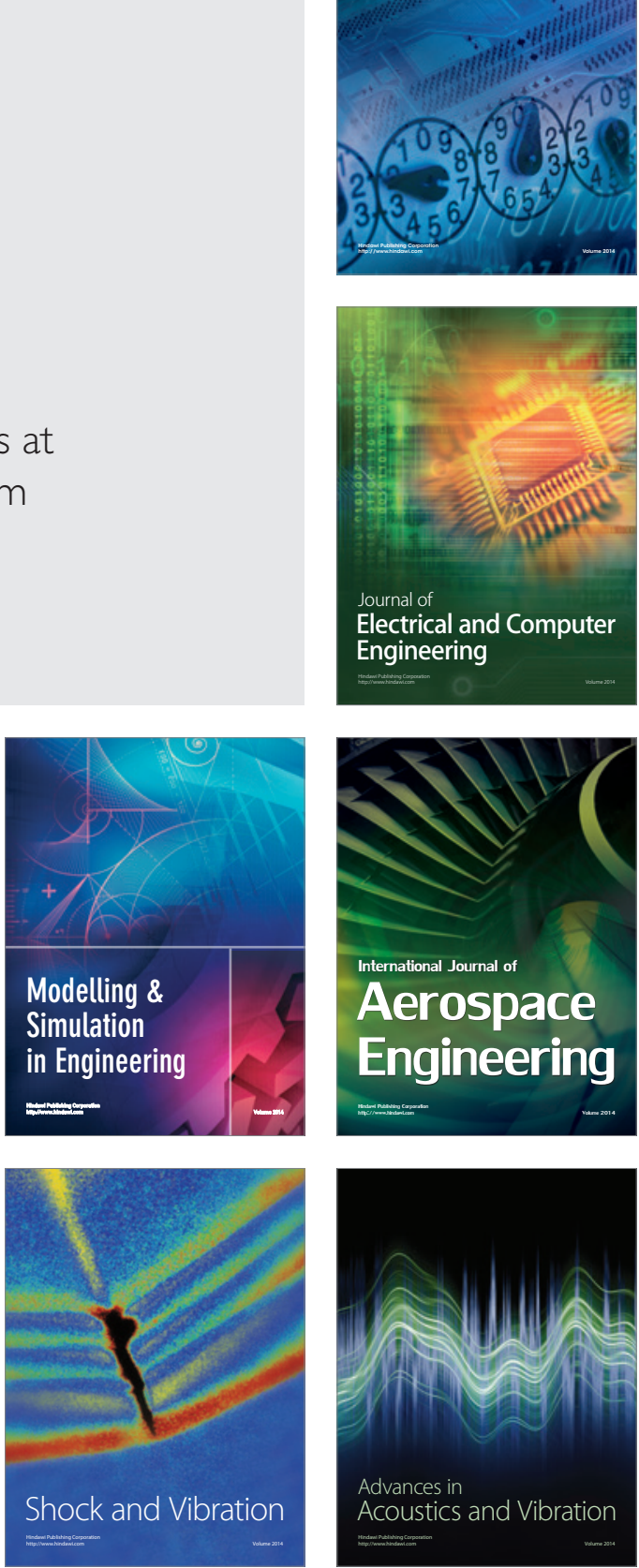